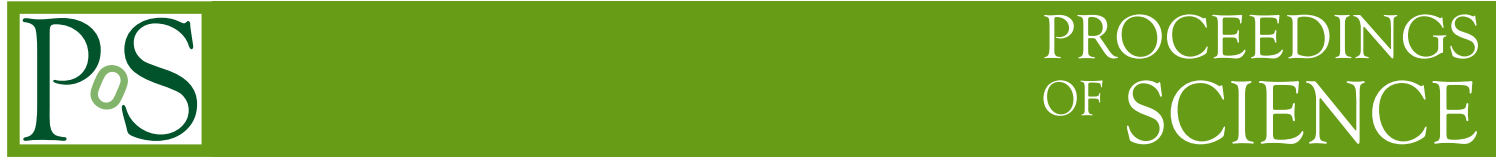

\title{
Measurements of top quark decay properties
}

\author{
Erich W. Varnes* \\ University of Arizona (USA) \\ E-mail: varnes@physics.arizona.edu
}

I report on measurements of the properties of the top quark using data collected by the CDF and DØ detectors during Run II of the Fermilab Tevatron. The helicity of the $W$ boson arising from top quark decay, the top quark decay branching ratios, and the top quark charge have all been measured, and the results in all cases are consistent with standard model expectations.

International Workshop on Top Quark Physics

January 12-15, 2006

Coimbra, Portugal

\footnotetext{
${ }^{*}$ Speaker.
} 


\section{Introduction}

The top quark was discovered during Run I of the Tevatron with about $60 \mathrm{pb}^{-1}$ of data [1,2]. With that sample it was clear that the top quark had a mass near $175 \mathrm{GeV}$, making it the only fermion with a Yukawa coupling near unity. This leads to speculation that non-standard model electroweak symmetry breaking mechanisms may be revealed through a detailed study of the properties of the top quark. In addition, such detailed studies would reveal the presence of an exotic component to the events being interpreted as $t \bar{t}$.

While the Run I sample was insufficient for the detailed measurement of the top quark charge, branching ratios, or decay vertex structure, we are in a much better position with Run II. Not only has the Tevatron's luminosity increased by over an order of magnitude, the center of mass energy has also been raised from 1800 to $1960 \mathrm{GeV}$, which increases the $t \bar{t}$ production cross section by about 30\%. In addition, both the CDF and D $\varnothing$ detectors have upgraded their capablities for top quark detection and measurement. Therefore we are now entering the era of precision measurements of the top quark's properties, the first set of which are reported here.

\section{Common features}

The analyses presented hear are based upon pair-produced top quarks, since single top production has yet to be observed. All use events in which one or both top quarks decay semileptonically, since these are easier to observe above background than the all-hadronic decay mode. In general the analyses are built upon event selection criteria used to measure the $t \bar{t}$ cross section, and may take advantage of the knowledge of the background levels gained in those analyses. Events in which one top quark decays semileptonically are called " $\ell+$ jets" events, while those in which both top quarks decay semileptonically are called "dilepton" events. Backgrounds in the $\ell+$ jets channel arise predominantly from $W+$ jets production or from QCD multijet production where one jet is misidentified as a lepton and missing $E_{T}$ arises due to mismeasurement of jet and lepton energies. In the dilepton channel backgrounds arise from several sources, notably $Z+$ jet production, with $Z \rightarrow \ell \ell$ or $Z \rightarrow \tau \tau \rightarrow \ell \ell$, and $W W$ or $W Z+$ jet production. The dilepton channel benefits from the small cross-section for background processes, but $t \bar{t}$ decays to the $\ell+$ jets channel are about six times more common, making the $\ell+$ jets channel the most powerful for measurements of top quark properties.

Systematic uncertainties arise from limited knowledge of the jet energy calibration and uncertainties in the modeling of signal and background events. In the event that the top quark mass is used as an input to the analysis, the uncertainty on that mass is also a systematic. Although most of the analyses presented here are statistically limited, it is important to note that many of the systematic uncertainties will not scale simply as $1 / \sqrt{N}$. This means that acheiving the ultimate precision on top quark properties measurements (i.e., during the LHC era) will require creativity and dedicated study to reduce the systematic uncertainties.

\section{Top quark branching ratio}

In the standard model the top decays to $W b$ with a branching fraction $>0.998$ at $90 \%$ C.L., constrained by the unitarity of the three-generation CKM matrix. However, if there are additional 

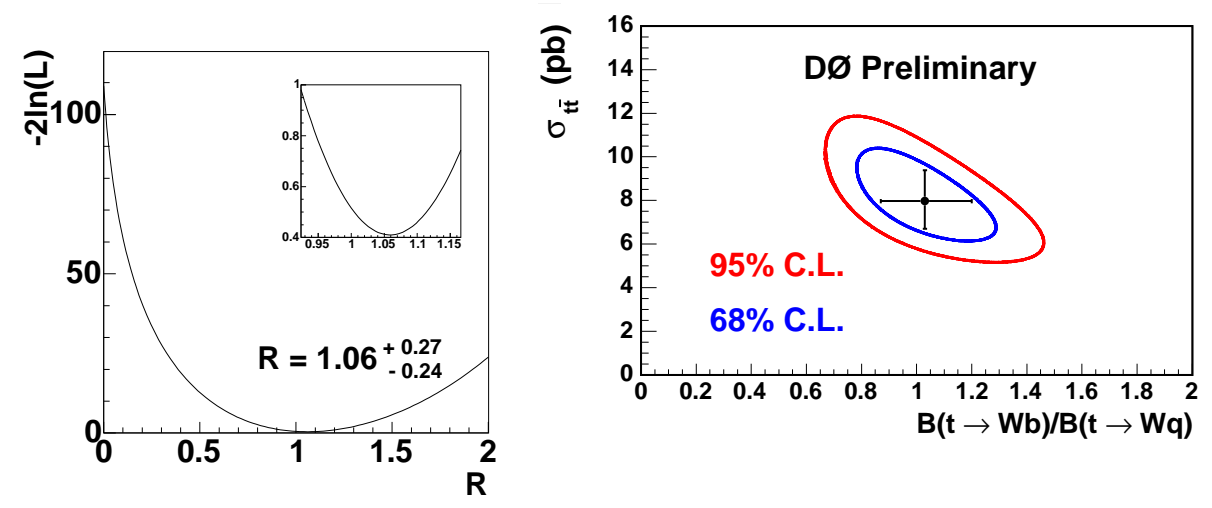

Figure 1: Results of measurements of $R$ (defined in the text) at CDF (left) and DØ (right). The standard model predicts $R=1$. The $\mathrm{D} \varnothing$ analysis performs a simultaneous measurement of $R$ and the $t \bar{t}$ production cross section.

quark generations this restriction would be lifted, allowing a greater rate of $t \rightarrow W q_{l}$ where $q_{l}$ is a $d$ or $s$ quark. This can be tested by using the finite $B$ hadron lifetime to identify jets arising from $b$ quarks and measuring the ratio:

$$
R \equiv \frac{B R(t \rightarrow W b)}{B R(t \rightarrow W b)+B R\left(t \rightarrow W q_{l}\right)}
$$

The distribution of jets identified as $b$ jets depends on $R$ as follows:

$$
\begin{aligned}
P_{n}(t \bar{t})= & R^{2} P_{n}(t \bar{t} \rightarrow b \bar{b}+X) \\
& +2 R(1-R) P_{n}\left(t \bar{t} \rightarrow q_{l} \bar{b}+X\right) \\
& +\left(1-R^{2}\right) P_{n}\left(t \bar{t} \rightarrow q_{l} \bar{q}_{l}+X\right)
\end{aligned}
$$

where $P_{n}(t \bar{t})$ is the overall probability to observe $n=0,1$ or 2 -tagged jets, $P_{n}(t \bar{t} \rightarrow b \bar{b}+X)$ is the probability to observe $n$ tagged jets when both top quarks decay to $W b, P_{n}\left(t \bar{t} \rightarrow q_{l} \bar{b}+X\right)$ is the probability to observe $n$ tagged jets when one top quark decays to $W b$ and the other decays to $W q_{l}$, and $P_{n}\left(t \bar{t} \rightarrow q_{l} \bar{q}_{l}+X\right)$ is the probability to observe $n$ tagged jets when both top quarks decay to $W q_{l}$. The $P_{n}$ depend upon the efficiencies for $b$ and light quark jets to satisfy the $b$ tagging criteria, which are measured in data control samples.

The distribution of $\ell+$ jets and dilepton events with zero, one, and two tagged $b$ jets in $160 \mathrm{pb}^{-1}$ of CDF data is consistent with standard model expectations, as shown in Table 1. A maximum likelihood fit to this distribution (shown in Fig. 1) yields $R=1.12_{-0.13}^{+0.21}$ (stat.) $\pm_{-0.13}^{0.17}$ (stat.) . This is equivalent to $R$ having a lower limit of 0.61 at $95 \%$ C.L. using the Feldman-Cousins technique.

$\mathrm{D} \emptyset$ performs a simultaneous measurement of the $t \bar{t}$ production cross section and $R$ by fitting the number of events with 0,1 , and $2 b$-tagged jets in $230 \mathrm{pb}^{-1}$ of data. The preliminary result of the fit is shown in Fig. 1. $\sigma_{t \bar{t}}$ is found to be $7.9_{1.5}^{+1.7}$ (stat. + syst.) \pm 0.5 (lumi.) pb, and $R$ is measured 


\begin{tabular}{ccccccc}
\hline & \multicolumn{3}{c}{$\ell+$ jets } & \multicolumn{3}{c}{ Dilepton } \\
& 0 tags & 1 tag & 2 tags & 0 tags & 1 tag & 2 tags \\
\hline Expected $S+B$ & $80.4 \pm 5.2$ & $21.5 \pm 4.1$ & $5.0 \pm 1.4$ & $6.1 \pm 0.4$ & $4.0 \pm 0.2$ & $0.9 \pm 0.2$ \\
Observed & 79 & 23 & 5 & 5 & 4 & 2 \\
\hline
\end{tabular}

Table 1: Distribution of tagged events in $160 \mathrm{pb}^{-1}$ of CDF data.

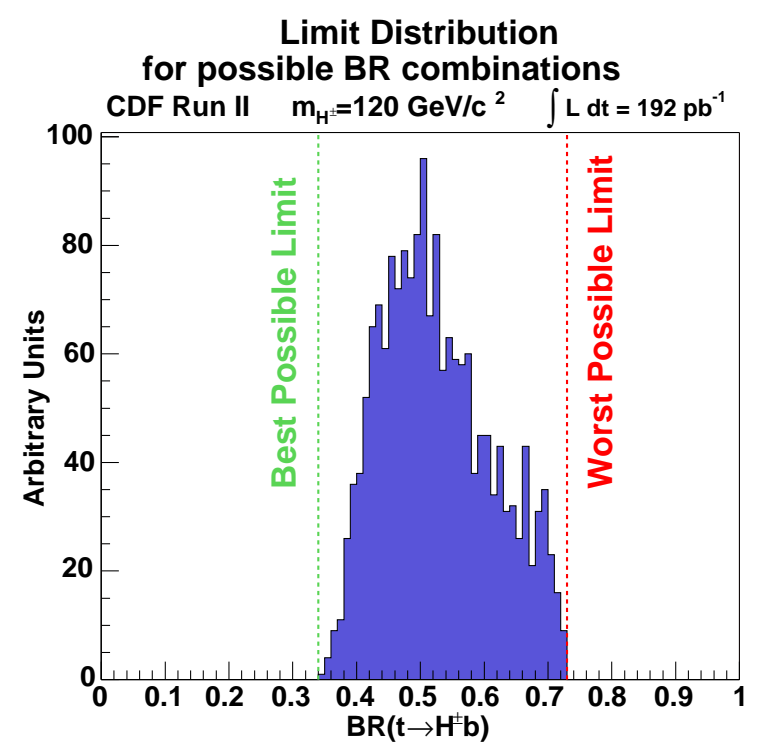

Figure 2: $95 \%$ C.L. upper limits on $B R\left(t \rightarrow H^{+} b\right)$ under various assumed $H^{+}$decay modes for a 120 $\mathrm{GeV} / c^{2} H^{+}$.

to be $1.03_{-0.17}^{+0.19}$ (stat. + syst.). Both values are consistent with standard model expectations, and using a Bayesian method the lower limit on $R$ is found to be 0.64 at $95 \%$ C.L.

Further details on the CDF measurement are available in Ref. [3], and the DØ measurement is described in Ref. [4].

In addition to the measurement of $R$, it is also important to search for evidence of $t \rightarrow H^{+} b$, which is allowed if there is a charged Higgs boson with mass less than that of the top quark. The standard model does not require a charged Higgs boson, so observing such a decay would indicate new physics. This is of particular interest because supersymmetry requires the existence of at least one charged Higgs boson.

The existence of the $H^{+} b$ decay mode would alter the distribution of $t \bar{t}$ modes, typically suppressing final states with electrons and or muons, and enhanching those with taus. Therefore sensitivity to $\mathrm{H}^{+} b$ decays requires the identification of $\tau$ leptons, which appear in the detector as a narrow jet of particles with a low multiplicity (typically one or three) of charged tracks.

The distribution of $t \bar{t}$ candidates in $192 \mathrm{pb}^{-1}$ of CDF data is consistent with standard model expectations, so there is no evidence of $t \rightarrow H^{+} b$. The upper limit that this implies for the branching ratio to $H+b$ depends upon both the $H^{+}$mass and decay branching ratios, which are model dependent. The limits obtained for a range of $H^{+}$decay models for a $120 \mathrm{GeV} H^{+}$are presented 
in Fig. 2. A full exploration of the constraints imposed on extensions to the standard model by this measurement is given in Ref. [5].

\section{4. $W$ boson helicity}

Given the measured top quark mass and the standard model $V-A$ structure of the $t W b$ vertex, the fraction of $W$ bosons from top quark decay with left-handed, longitudinal, and righthanded polarizations are predicted to be $f_{0}=0.70, f_{-}=0.30$, and $f_{+}=\mathscr{O}\left(10^{-4}\right)$, with uncertainties due to higher-order corrections of order $1 \%$. Therefore a measurement that disagrees with these values would be a clear sign of physics beyond the standard model. For example, a $V+A$ term in the $t W b$ coupling would increase $f_{+}$but leave $f_{o}$ unchanged.

Any leptonic top quark decay can be used to measure the $W$ boson's helicity. There are two observables that are sensitive to the $W$ boson helicity. The first is the helicity angle $\theta^{*}$, defined as the angle in the $W$ boson rest frame between the charged lepton and top quark directions. To measure this angle, one needs to determine which of the jets in the event should be paired with the lepton. In the $\ell+$ jets decay channel, this is done using a constrained kinematic fit to the $t \bar{t}$ hypothesis, with the known top quark mass $(175 \mathrm{GeV})$ providing an additional constraint. The fit is performed using all twelve possible jet assignments (six if the event has a single $b$-tagged jet that is assumed to not arise from $W$ decay, two if there are two such $b$-tagged jets), and the combination that yields the lowest $\chi^{2}$ for the fit is chosen. In untagged events, this procedure selects the correct $b$ jet about $60 \%$ of the time. Once this is done, one can either boost to the reconstructed $W$ boson rest frame and calculate $\theta^{*}$, or one can use the approximation:

$$
\cos \theta^{*} \approx \frac{2 m_{\ell b}^{2}}{m_{t}^{2}-m_{W}^{2}}-1
$$

$\mathrm{D} \varnothing$ uses the former approach while CDF chooses the latter. Left-handed $W$ bosons will tend to have $\cos \theta^{*}$ near -1 , longitudinal $W$ bosons will prefer values near 0 , and right-handed $W$ bosons will prefer $\cos \theta^{*}$ near +1 .

A simpler approach is to note the transverse momentum of the lepton in the laboratory frame, since left-handed $W$ bosons will tend to emit the lepton opposite to the $W$ boson flight direction, and the opposite is true for right-handed $W$ bosons. This variable is less sensitive than $\cos \theta^{*}$, but is more readily applicable to dilepton events and $\ell+$ jets in which only three of the four final-state jets are reconstructed. Each dilepton event provides two measurements of lepton $p_{T}$, which partially compensates for the small $t \bar{t}$ branching ratio to dileptons.

Once events are selected and the $W$ helicity variable is recorded, the distribution in data is compared to the expectations from background and signal with various $W$ helicities using a binned maximum likelihood fit. The $\ell+$ jets and dilepton channels are used by both $\mathrm{CDF}$ and $\mathrm{D} \emptyset$ and the results are presented in 4 for CDF and Fig. 5 for DØ. CDF finds [6]:

$$
\begin{aligned}
& \left.f_{o}=0.99_{-0.35}^{+0.29} \text { (stat. }\right) \pm 0.19 \text { (syst.) }(\ell+\geq 4 \text { jets }) \\
& \left.f_{o}=0.31_{-0.23}^{+0.37} \text { (stat. }\right) \pm 0.17 \text { (syst.) }(\ell+\geq 4 \text { jets plus dileptons }) \\
& f_{o} \quad=0.70_{-0.34}^{+0.22} \text { (stat.+syst. } \quad \text { (combined) }
\end{aligned}
$$



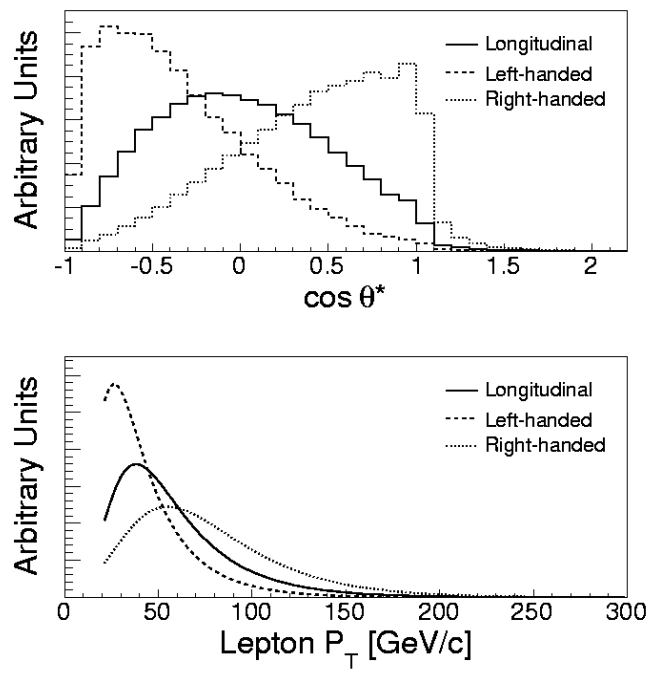

Figure 3: Monte Carlo distributions of $\cos \theta^{*}$ (left) and lepton $p_{T}$ (right) for the three $W$ helicity states.
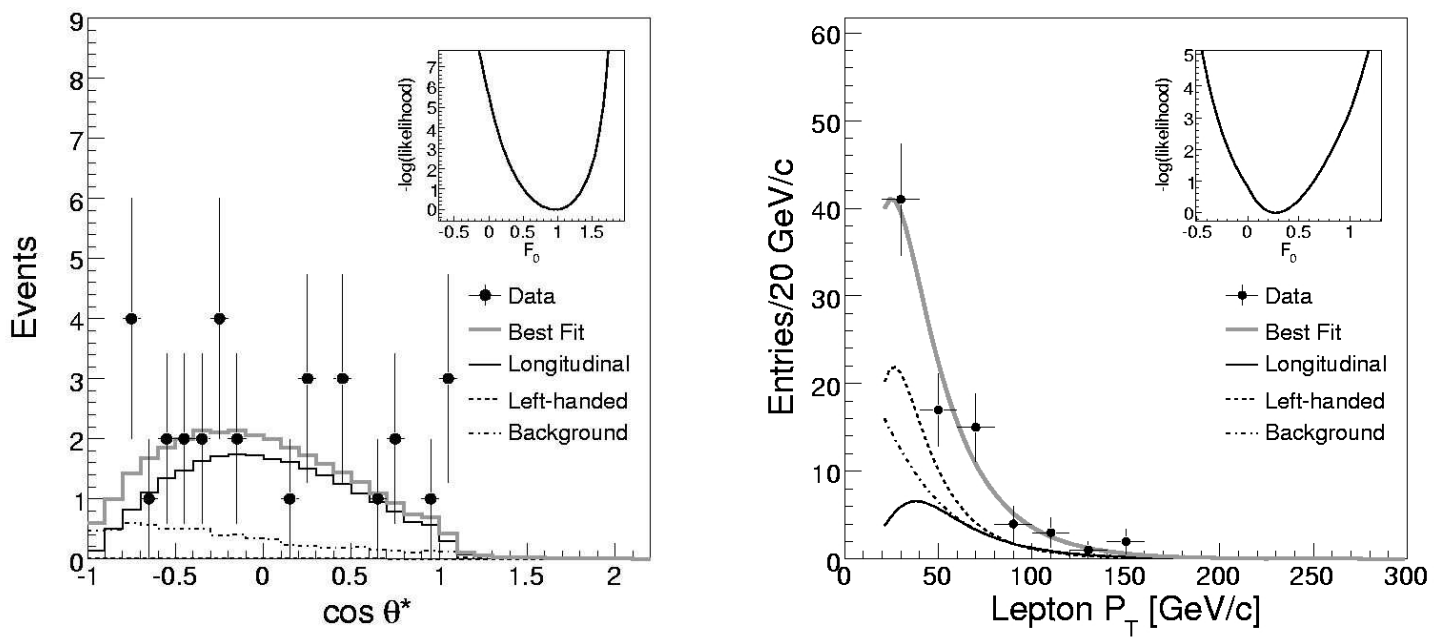

Figure 4: Fraction of longitudinally-polarized $W$ bosons observed at CDF using $\cos \theta^{*}$ (left) and lepton $p_{T}$ (right).

$$
f_{+} \quad=0.00_{-0.19}^{+0.20}(\text { stat.+syst. } \quad \text { (combined) }
$$

$\mathrm{D} \emptyset$ uses $230 \mathrm{pb}^{-1}$ of $\ell+$ jets data to measure [7]

$$
f_{+}=0.00 \pm 0.13 \text { (stat.) } \pm 0.07 \text { (syst.) }
$$

and $370 \mathrm{pb}^{-1}$ of dilepton data to preliminarily measure [8]

$$
f_{+}=0.13 \pm 0.20 \text { (stat.) } \pm 0.06 \text { (syst.). }
$$



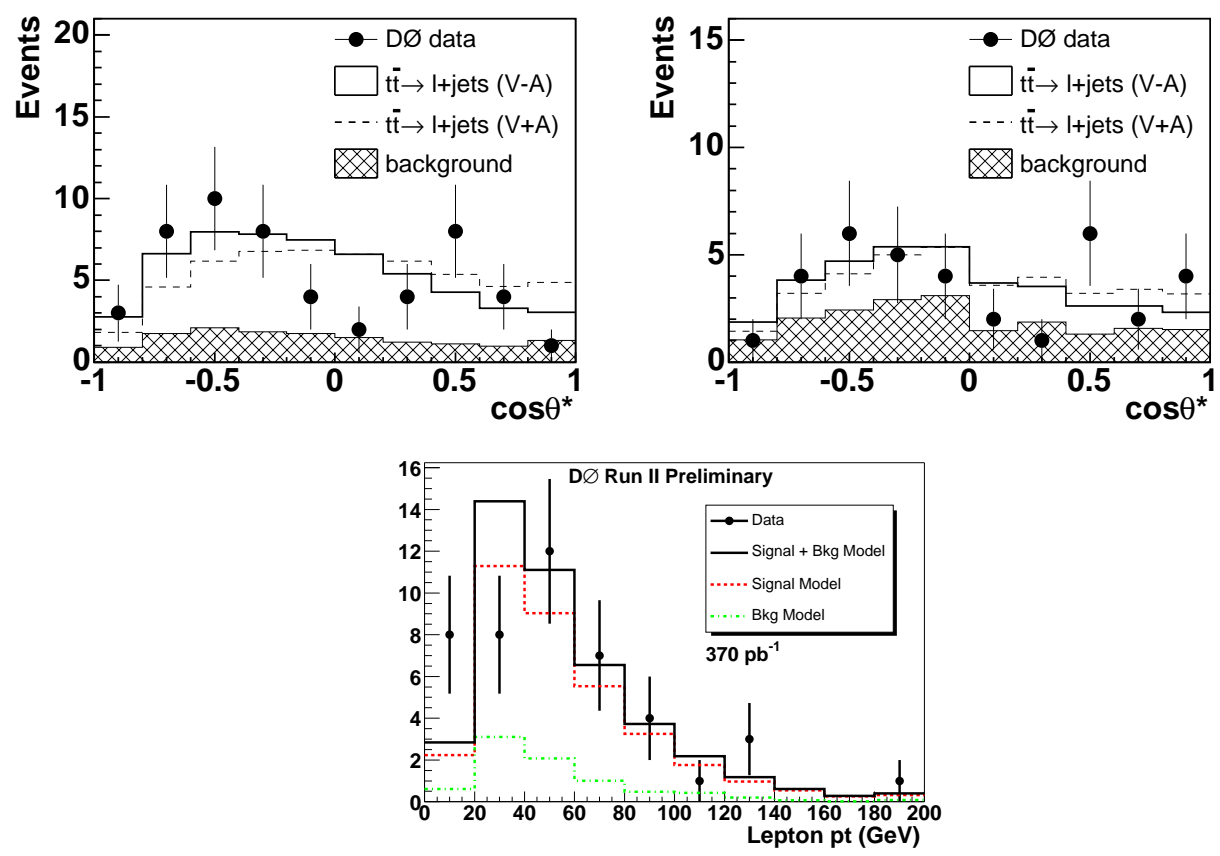

Figure 5: Comparison of $\mathrm{D} \emptyset$ data to expectations $V-A$ and $V+A t W b$ couplings in $b$-tagged $\ell+$ jets events (top left), untagged $\ell+$ jets events (top right) and dilepton events (bottom).

The combination of these results (which is also preliminary) yields

$$
f_{+}=0.04 \pm 0.11 \text { (stat.) } \pm 0.06 \text { (syst.) }
$$

which corresponds to a $95 \%$ Bayesian upper limit of 0.25 .

All of these measurements are consistent with the standard model.

\section{Top quark charge}

In the standard model the top quark is defined as having charge $+2 / 3$. However, in certain extensions there is an additional charge $-4 / 3 t^{\prime}$ quark with production and decay properties similar to the top quark. If nature is described by such a theory, and the top quark is too heavy to have been observed at the Tevatron, the particle we've been exploring may in fact be the $t^{\prime}$. We can distinquish between the two scenarios by summing the charges of the top quark's decay products, which is done most easily with semileptonic decay modes. Note that since there is a top and antitop quark in each event, with no way to distinguish between them without assuming a charge sign, we are only sensitive to the absolute value of the top quark charge. To measure the top quark charge one needs to select the $b$ jet to pair with the lepton. The problem is simplified by requiring the event to have two $b$-tagged jets, thereby reducing the number of possible jet assignments from twelve to two. The pairing that fits to the $t \bar{t}$ hypothesis with smallest $\chi^{2}$ is selected. The lepton charge is measured from the curvature of the charged track, but measuring the charge of the $b$ presents a challenge since the $b$ appears as a jet of final-state particles. By taking a $p_{T}$-weighted sum of the charges of tracks within the $b$ jet's cone, one can distinguish $b$ from $\bar{b}$ on a statistical basis. The 


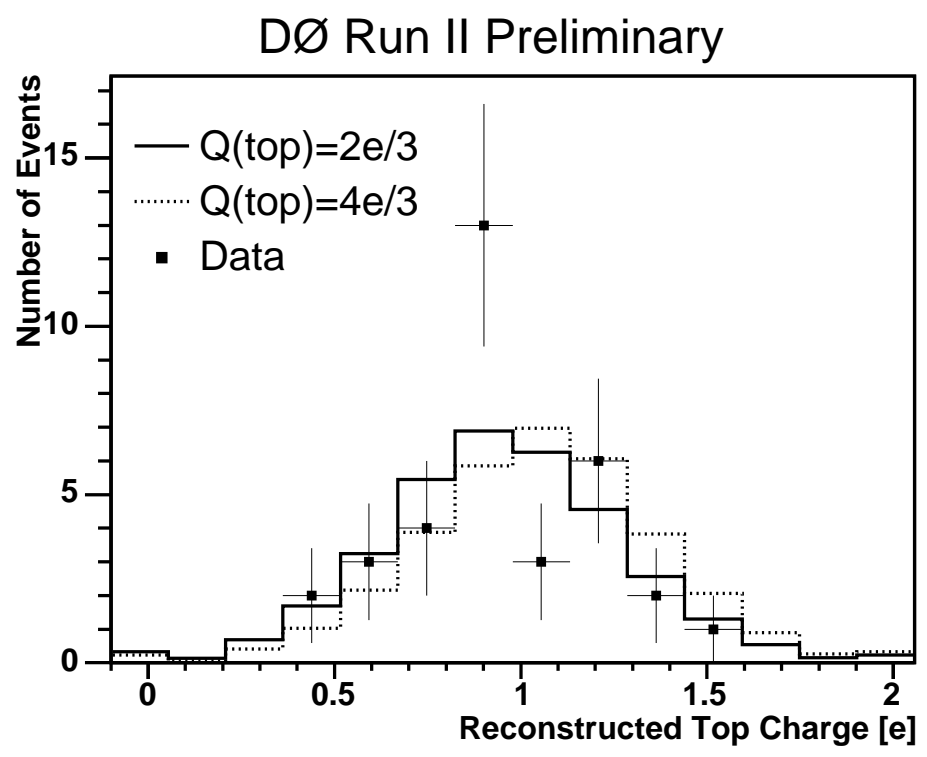

Figure 6: Distribution of reconstructed top quark charge observed in data (points with errors) and expectations for charge with absolute value $2 / 3 e$ (solid histogram) and $4 / 3 e$ (dashed histogram).

performance of this jet-charge algorithm is tested using dijet data in which both jets are vertextagged and one also has a muon within the jet cone to tag the charge of one of the $b$ jets. The performance of the jet charge algorithm is calculated as a function of the $\eta$ and $p_{t}$ of the jet, so that the kinematic differences between the control sample and the $t \bar{t}$ are accounted for.

The reconstructed charge observed in $t \bar{t}$ candidates in $370 \mathrm{pb}^{-1}$ of $\mathrm{D} \emptyset$ data is presented in Fig. 6. The data favors the standard model hypothesis, and the $-4 / 3 e$ scenario is preliminarily ruled out at 94\% C.L. More details on this measurement are available in Ref. [9].

\section{Summary}

Several properties of the top quark have been measured in Run II of the Tevatron, including its decay branching ratios, charge, and the helicity of the $W$ boson arising from its decay. These measurements, based on a few hundred $\mathrm{pb}^{-1}$ of data, are all consistent with standard model expectation. Many, however, are limited by statistics, so the rapidly increasing Run II dataset will allow more incisive measurements to be made in the near future. Detailed examination of the nature of the top quark will continue to be one of the primary goals of the Run II physics program.

\section{References}

[1] F. Abe et al. (CDF Collaboration), Phys. Rev. Lett. 74, 2626 (1995).

[2] S. Abachi et al. (DØ Collaboration), Phys. Rev. Lett. 74, 2632 (1995).

[3] D. Acosta et al. (CDF Collaboration), Phys. Rev. Lett. 95102002

[4] DØ Collaboration, DØ note 4833-CONF 
http://www-d0.fnal.gov/Run2Physics/WWW/results/prelim/TOP/T14/T14.pdf

[5] A. Abulencia et al. (CDF Collaboration), hep-ex/0510065, to be published in Phys. Rev. Lett.

[6] A. Abulencia et al. (CDF Collaboration), hep-ex/0511023, submitted to Phys. Rev. Lett.

[7] V.M. Abazov et al. (DØ Collaboration), Phys. Rev. D 72, 011104(R) (2005)

[8] DØ Collaboration, DØ note 4839-CONF

http://wWw-d0.fnal.gov/Run2Physics/WWW/results/prelim/TOP/T16/T16.pdf

[9] DØ Collaboration, DØ note 4876-CONF

http://wWW-d0.fnal.gov/Run2Physics/WWW/results/prelim/TOP/T19/T19.pdf 